\title{
Acoustic Filtration, Fractionation, and Mixing in Microfluidic Systems
}

\author{
A. Wang, K. Fisher
}

February 4, 2002

U.S. Department of Energy

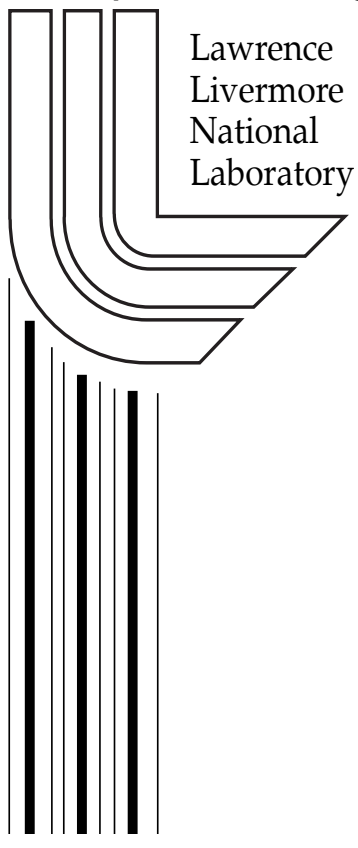




\section{DISCLAIMER}

This document was prepared as an account of work sponsored by an agency of the United States Government. Neither the United States Government nor the University of California nor any of their employees, makes any warranty, express or implied, or assumes any legal liability or responsibility for the accuracy, completeness, or usefulness of any information, apparatus, product, or process disclosed, or represents that its use would not infringe privately owned rights. Reference herein to any specific commercial product, process, or service by trade name, trademark, manufacturer, or otherwise, does not necessarily constitute or imply its endorsement, recommendation, or favoring by the United States Government or the University of California. The views and opinions of authors expressed herein do not necessarily state or reflect those of the United States Government or the University of California, and shall not be used for advertising or product endorsement purposes.

This work was performed under the auspices of the U. S. Department of Energy by the University of California, Lawrence Livermore National Laboratory under Contract No. W-7405-Eng-48.

This report has been reproduced directly from the best available copy.

Available electronically at http://www.doc.gov/bridge

Available for a processing fee to U.S. Department of Energy

And its contractors in paper from

U.S. Department of Energy

Office of Scientific and Technical Information

P.O. Box 62

Oak Ridge, TN 37831-0062

Telephone: (865) 576-8401

Facsimile: (865) 576-5728

E-mail: reports@adonis.osti.gov

Available for the sale to the public from

U.S. Department of Commerce

National Technical Information Service

5285 Port Royal Road

Springfield, VA 22161

Telephone: (800) 553-6847

Facsimile: (703) 605-6900

E-mail: orders@ntis.fedworld.gov

Online ordering: http://www.ntis.gov/ordering.htm

\section{OR}

Lawrence Livermore National Laboratory

Technical Information Department's Digital Library

http://www.llnl.gov/tid/Library.html 


\section{Table of Contents}

$\begin{array}{lll}1.0 & \text { Overview } & 3 \\ 1.1 & \text { Objectives } & 3\end{array}$

1.1 Objectives

1.2 Approach 3

2.0 Background 4

2.1 Acoustic particle manipulation (theory) 4

2.2 Mixing 5

2.3 Filtration/Fractionation (particle focusing) 6

3.0 Modeling 8

3.1 One-dimensional layered transmission model 8

3.2 Application of commercial finite element package (ABAQUS) 11

4.0 Hardware development 13

4.1 Standing chamber (cuvette) application 13

4.1.1 One-dimensional transmission matrix approximation 14

4.1.2 Two-dimensional ABAQUS finite element model 15

$\begin{array}{ll}\text { 4.1.3 Mixing (enhanced binding) } & 17\end{array}$

4.2 Flow-through device (flocel) application 18

4.2.1 Holding/Filtering of particles in flowing medium 20

5.0 Commercialization of selected results 21

$\begin{array}{ll}\text { 6.0 Summary } & 22\end{array}$

$\begin{array}{lll}\mathbf{7 . 0} & \text { References } & 23\end{array}$ 


\subsection{Overview}

\subsection{Objective}

This project is concerned with the research and development of a technique to manipulate small particles using acoustic energy coupled into a fluid filled plastic or glass sample chamber. These resulting miniaturized systems combine high functionality with an inexpensive, disposable sample chamber.

\subsection{Approach}

Our approach to this problem is based on a combination of sophisticated modeling tools in conjunction with laboratory experiments. The design methodology is summarized in Figure 1. The process begins by investigating a wide range of device parameters using a one-dimensional analytical approximation. The results of these initial parameter studies are incorporated into a sophisticated three-dimensional multi-physics finite element code. From these simulations the optimized designs are prototyped and experimentally tested. The results of the experimental observations are then used to improve analytical approximations and the process is repeated as necessary.

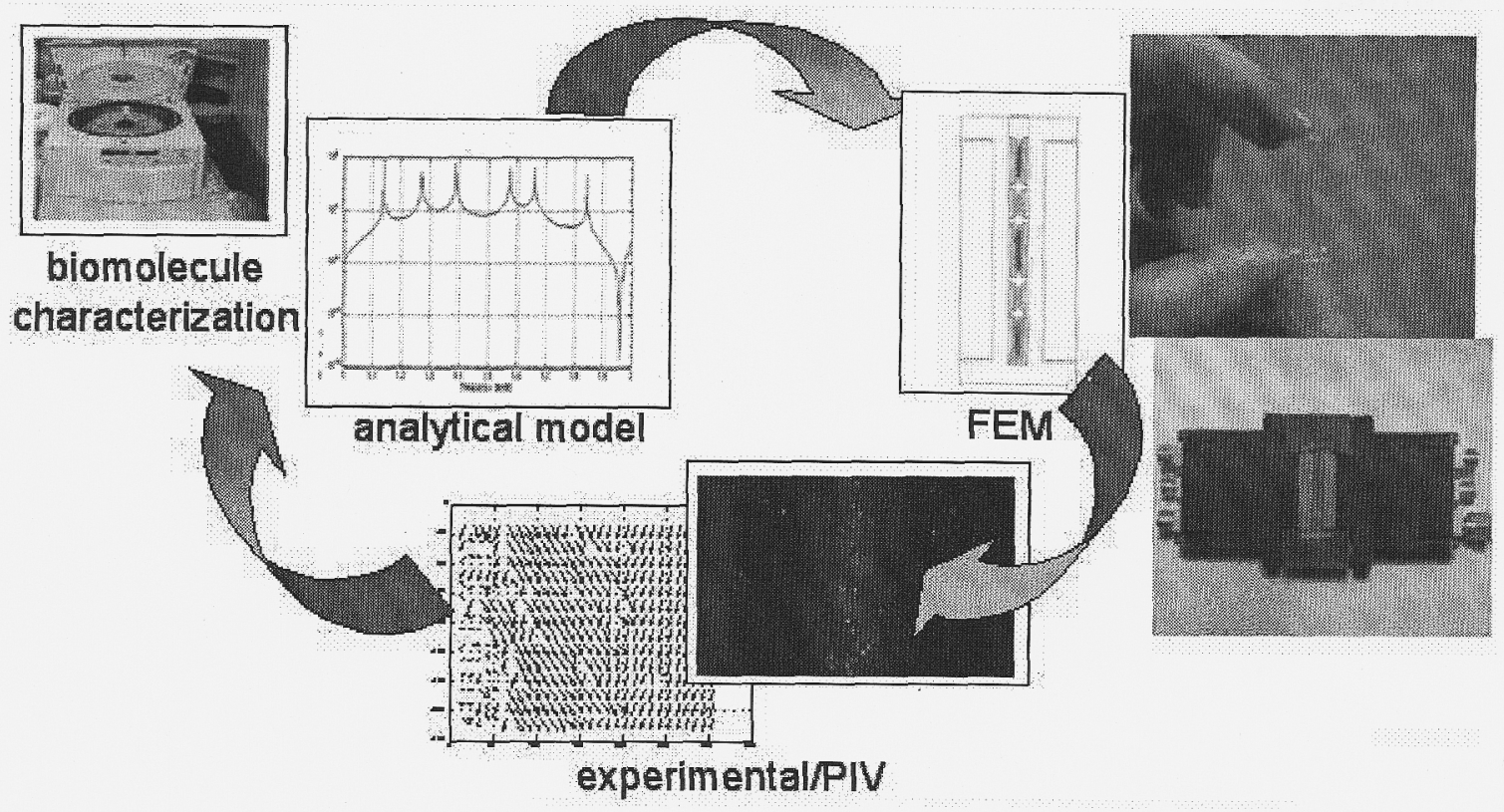

Figure 1. The design methodology is based on, analytical, computational, and experimental methods. 


\subsection{Background}

The threat of chemical and biological warfare agents has made apparent the need for autonomous, portable, and fast pathogen detection systems. Sample pre-treatment is one of the most critical aspects in the development of autonomous systems, yet it remains largely an unsolved problem. Sample handling is also of key interest in private industry. The global market for microelectromechanical systems (MEMS) is currently estimated at $\$ 10$ billion, with biological and medical MEMS representing the most rapidly growing sector. The medical arena brings the additional challenges of disposability and low cost. Emerging MEMS-based companies, such as Affymetrix, Caliper, and ACLARA, are targeting miniaturized microfluidic systems that are able to perform a complete bioassay on what is loosely defined as a "chip." A necessary goal of these systems is to obviate manual handling of samples, which is slow, requires trained personnel, and is not cost-effective. Integrated sample preparation requires a high degree of functionality to truly permit biological or chemical analysis to be performed in a miniaturized system, yet the danger of sample-to-sample contamination dictates inexpensive, disposable parts. Typically the cost of a part increases with its functionality.

Acoustic-based systems provide the ability to perform basic fluidic functions necessary in an analysis system, specifically filtration, fractionation, concentration, and mixing. Since the actuation method depends upon acoustic energy coupled in from an external source, integration of the piezoelectric transducers with the chamber is not required. Hence, glass or plastic fluidic chambers can be manufactured inexpensively and thrown away after one use, while the piezoelectric transducers and associated electronics are reused. In addition, the electronics of acoustically-driven systems are isolated from the biological samples, reducing the likelihood of electrode fouling. This will increase the lifetime of the system, reduce maintenance, and permit continuous monitoring. The elimination of integrated components also allows the layout of the microfluidic system to be more easily reconfigurable and amenable to rapid prototyping.

Ultrasonic manipulation of particles in fluidic suspensions has been demonstrated in numerous examples in the literature, Whitworth [1], provides an excellent $\mathrm{r}$ overview of some of the more recent developments in large macro-scale fluidic systems (i.e. with length scales larger than $1 \mathrm{~mm}$. Numerous other authors have also demonstrated various techniques and devices to manipulate particles such as cell harvesting [2], concentrating Erythrocytes [3] and transport [4]. However, from a microfluidics standpoint, acoustic transport mechanisms represent a new area of research. The current emphasis of this research effort was to apply these acoustic transport mechanisms to sample pretreatment or preparation. Particular interest was in mixing and focusing of small 1-10 um particles. Ultrasonic mixing brings with it the advantage of rapid, mixing, no moving parts, and no need for external injection of fluids or nozzles to create turbulence. The ability to mix samples in a small flow through chamber will speed the binding time required for immunoassays, increasing the throughput and speed of the system. The same acoustic force used to mix the particles in the chamber can, with the appropriate design considerations, be used to focus and hold the particles against an ambient flow. This has considerable utility for biological applications where specific bead based binding assays require binding and rinsing steps. Enhancing binding through focusing has the potential to reduce the actual interaction volume from the entire sample chamber to specific nodal binding zones (acoustic field nodes in the standing wave chamber). These nodal binding zones also have the dual purpose of acting as holding zones for dissimilar beads or particles to improve affinity binding processes and retain the particles against the ambient flow of the host fluid or reagent.

\subsection{Acoustic particle manipulation (theory)}

Our approach is to use acoustic energy coupled into plastic fluid channels via bulk piezoelectric transducers. Distinct transport mechanisms can be activated by varying the orientation of the 
piezoelectric transducer and chamber configurations, or by changing the input energy levels. The primary acoustic transport principles are acoustic radiation pressure and acoustic streaming. The acoustic radiation pressure was first analyzed by King [5], who reported that particles moved to the nodes or antinodes of a standing acoustic wave depending on the relative density between the particle and the suspending liquid. Yosioka and Kawasima [6] reported the dependence of the radiation pressure on the compressibility of the particles, introducing the concept of a "compressdensity" factor. The basic physics relies on the fact that the acoustic radiation pressure exerts a force on a particle. Radiation pressure generated in the presence of a standing wave induces a force field that accelerates particles towards the nodes or anti-nodes of the standing wave. The acoustic force exerted on a particle can be expressed as,

$$
F_{a c}=\frac{4 \pi}{3} r^{3} E_{a c} A(\rho, \lambda) \sin (2 k x)
$$

where, $r$, denotes the particle radius, $\mathrm{k}$ is the wave number, and $\mathrm{E}_{\mathrm{ac}}$ is the acoustic energy density. The compressidensity factor, $A(\rho, \lambda)$, reflects the relative density, $\rho$, and compressibility, $\lambda$, (i.e., $1^{\text {st }}$ lame constant) of the elasticity of particle with respect to the ambient medium. Finally, the sign of A determines whether the particles concentrate in the nodes or the anti-nodes of the standing wave [7]. It follows that acoustic radiation pressure could potentially be used to differentiate particles based on their size and their material attributes.

\section{Radiation Pressure Force:}

$\mathrm{Fac}=-\mathrm{V}_{0} \mathrm{Ek} A(\rho, y) \sin (2 \mathrm{kx})$

$V_{0}$ - particle volume

E- energy density

$k$ - wave number

A - compressidensity factor

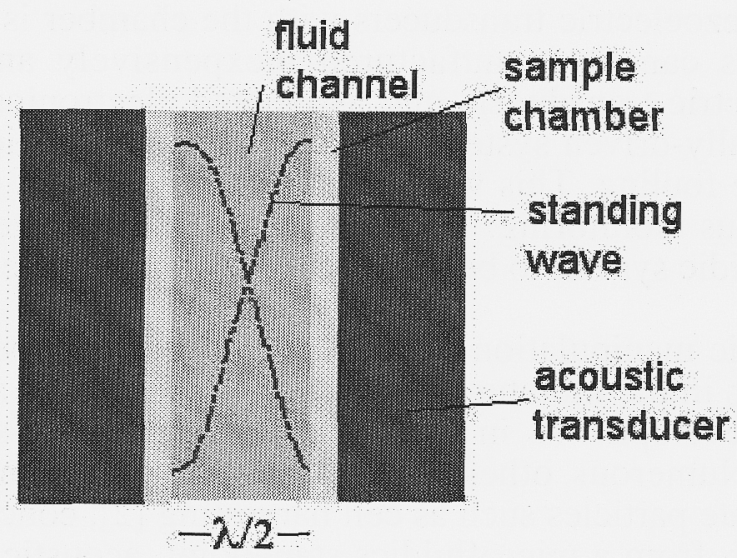

Figure 2. Particles move to the nodes or antinodes of a standing acoustic wave depending on their material properties.

\subsection{Mixing}

The ability to mix fluids is a necessary capability for integrated sample preparation. Micro-scale mixing is a challenge since small channel dimensions make it difficult to create turbulence. The immunoreaction relies upon diffusion of affinity partners to the surface of the solid-phase, such as a fluorescent beat, and can be limited by the rate of mass transport. It follows that if the rate of mass transport can be increased this can speed up the rate of binding, ultimately increasing throughput and decreasing response time of the system. Studies have been performed that compare the effect of mechanical shaking and ultrasonic mixing on antibody-antigen binding [89]. In these studies, results show that the amount of bound hormone in their assay increased linearly with acoustic power. It was also found that the reaction rate constant increased to as much as 500-fold from that of samples that were not exposed to ultrasound during incubation periods. Although the mechanism for increased affinity binding is not well understood, it is hypothesized that a combination of increased temperature and mass transport results in more rapid affinity binding than is achievable via those mechanisms alone. Ultrasonic mixing can be 
produced by either acoustic streaming [10] or acoustic radiation pressure. Acoustic streaming is a nonlinear phenomenon that results in net fluid motion due to a high-energy acoustic field. The amount of motion (or mixing) is a function of the acoustic field. Mixing can also arise from acoustic radiation pressure acting on individual particles in a fluidic system. Imbalances in the radiation pressure within the system can induce a mixing effect.

Acoustic mixing and particle transport can be accomplished by driving two acoustic transducers in an imbalanced manner, so that a standing wave is not set up. Subtle differences in drive frequency or phase result in a slowly-traveling standing wave that can be used to transport particles. Similar mixing effects can be achieved by operating a single transducer and a reflector. This arrangement simplifies the design and eliminates any inherent differences between driving elements. Mixing can be initiated by a single transducer if it is driven at a frequency that creates an 'unstable' standing wave pattern. Unstable standing wave patterns are obtained by selecting a drive frequency that is equal to, or near, two or more eigen modes of the standing wave chamber. The two modes will essentially beat against on another creating large spatially dependent radiation pressure imbalances and significant mixing. Higher-intensity fields can be used to produce acoustic streaming if motion in the liquid is desired.

\subsection{Filtration/Fractionation (particle focusing )}

In contrast to acoustic streaming, which acts upon the fluid, acoustic radiation pressure exerts a force on a particle in the fluid. This force is a function of the size, density, and compressibility of the particle with respect to the surrounding liquid. Radiation pressure generated in the presence of a standing wave induces a pressure field that causes particles to collect in the nodes or antinodes of the standing wave. The acoustic force was described in Section 2.1. Figures 3 and 4 show two approaches to achieving particle filtration, concentration, and density fractionation using the acoustic radiation pressure.

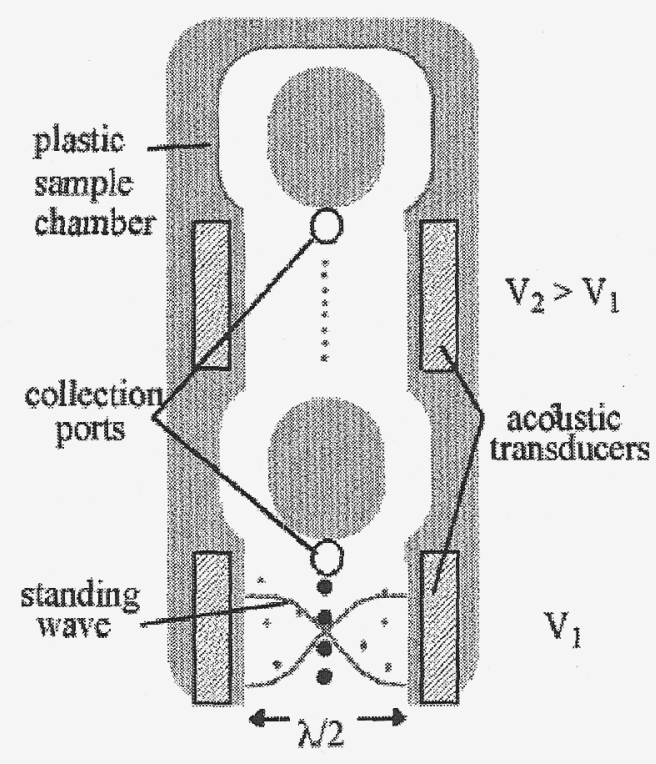

Fig 3 Top wiew of acoustic concentrator, filter, and fractionator.

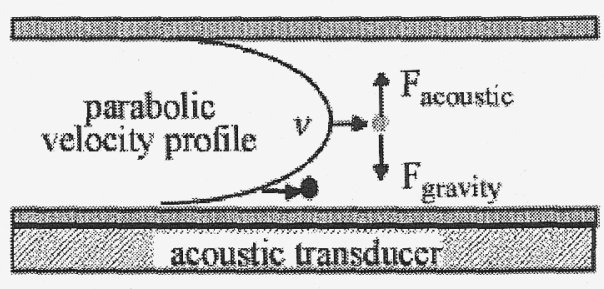

Fig 4 Side view of density fractionator

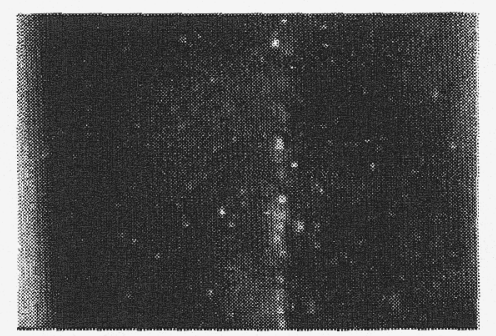

Fig 5 Preferential concentration of $10 \mathrm{um}$ over $3 \mu \mathrm{m}$ beads at the center of a fluid channel. 
Figure 3 shows a device that can be used for concentration, filtration and fractionation of particles. Acoustic transducers are placed on either side of a microfluidic channel with a width equal to half the wavelength of the acoustic wave. When the transducers are driven 180 degrees out of phase, a standing wave is generated in the channel causing particles to concentrate in the pressure node generated at the center of the channel. Acoustic energy can be controlled such that larger, more dense (black) particles are captured and filtered by the radiation pressure, allowing smaller, lighter (gray) particles to pass. If more than one of these configurations is placed in series, particles traverse a set of bifurcations of increasing acoustic energy (voltage applied to the acoustic transducers). Particles of higher density or larger size will be captured first at lower energy densities and removed through a collection port. As particles traverse higher energy bifurcations, smaller, lighter particles are sequentially collected and fractionation is accomplished.

Fiigure 4 shows a device that uses acoustic levitation by balancing the acoustic radiation pressure force against the gravitational force. The concept is akin to typical fractionation techniques, which make use of the parabolic velocity field in a channel to separate out particles that are positioned at varying heights in the channel. Particles that are levitated to the center of the channel will be expelled from the channel more rapidly than those close to the wall.

Although the basic design of an acoustically driven microfluidic mixer or fractionator is extremely simple, accurately controlling the standing wave pattern is not. This complexity warrants sophisticated multi-physics models incorporating piezoelectric, structural, and fluidic effects to predict system performance. To this end, computational modeling techniques were applied to understand and quantify the physical phenomena associated with these microfluidic devices. The next section presents two approaches that have been used extensively to model the performance of acoustically driven microfluidic devices. 


\subsection{Modeling devellopment}

The acoustically driven microfluidic systems described in the previous chapter all involve the transport and manipulation of beads and target species or pathogens. As discussed above, the transport and predictable manipulation of the desired constituents is of critical importance to the device functionality. The proposed devices cited above require a deeper understanding of the fundamental forces at play in the system. Furthermore, because the length scales of the channels used to transport, mix, and fractionate are so small in microfluidic systems (e.g., microns to millimeters), intermolecular forces that are typically masked in bench-scale instruments also play an important part in the target species behavior during transport and manipulation processes. To augment and guide the device design, several research approaches have been employed to study the behavior of the target species subject to the complex interplay of the force interactions on the particles. LLNL is currently involved with a research effort focused on developing a new threedimensional discrete simulation capability, which is based on the Boltzmann equation [11, 12] has been developed under the LLNL research and development program (LDRD) that permits the study of stationary and mobile macro-particles in microfluidic devices. This work is currently being extended to incorporate intermolecular force interactions. The simulation capability readily permits the inclusion of new physics. In particular, the effect of acoustic forces on the particles can be studied by the inclusion of pressure fields from the standing wave patterns induced form the bulk piezoelectric drivers. Therefore, the scope of the numerical models presented in this research has been limited to developing a comprehensive picture of the acoustic fields generated in a coupled fluid-elastic structure. Fluid viscosity, ambient flow, and inertial effects of the particle are not included. Even with these simplifications, the inherent complexity of even a simple one-dimensional system requires a multi physics approach relating piezoelectric, structural, and acoustic effects into a single numerical model. The key parameter in these models is the acoustic energy density which is given as

$$
E_{a c}=\frac{1}{2} \rho_{o}\left(u^{2}+\frac{p^{2}}{\rho_{o}^{2} c^{2}}\right)
$$

Here $u$ and $p$ are the acoustic pressure and acoustic velocity, $\rho_{o}$ is the fluid density, and $c$ is the speed of sound in the fluid. The corresponding radiation force on a particle is directly proportional to this quantity (refer to $\mathrm{Eq}(1)$ ) and thus serves as a design parameter for investigating operational ( frequency, drive voltage), geometrical (channel, wall, driver thickness) and material properties specific to a given design. The motivation here is to couple as much acoustic energy into the fluid as possible whereby maximizing particle manipulation capabilities and control.

\subsection{One-dimensional layered transmission model}

The predictable manipulation of beads and target species or pathogens in millimeter scale fluidic devices has relied heavily on empirical techniques and simple geometrical arguments to guide the device construction and operation [1,3]. In many cases the devices are designed to approximate planar one-dimensional systems. Under these conditions, it is possible to establish a robust standing wave field over a large number of discrete frequencies. However, very little work has been done in the area of modeling the structural acoustic response of the standing wave pattern in the fluid as a function of the drive parameters on the bulk piezoelectric transducers. Recall form Eq. (1), that the radiation force on a given particle is directly proportional to the 
acoustic energy density in the standing wave field. This energy density is in turn directly related to the pressure and the acoustic particle velocity described by Eq. (2).

A one-dimensional harmonic model based on a layered transmission matrix method is now presented to determine the acoustic energy density in the fluid channel by calculating the pressure and velocity profiles in the fluid channel. In this model, the transmission matrix relates the normal stress (pressure) and velocity at each interface of the one dimensional layered system to a drive voltage applied to a piezoelectric layer. The utility of this one-dimensional model is that it can be used to guide the design by optimizing performance with respect to frequency, geometry and material properties. Numerous models are available in the literature that address a similar problem of determining the transmission and reception efficiency of an underwater electro acoustic transducer [13-15]. We will modify an approach initially developed by Lewis $[15]$, to our specific application. Figure 2 shows the layered geometry that will direct the model development.

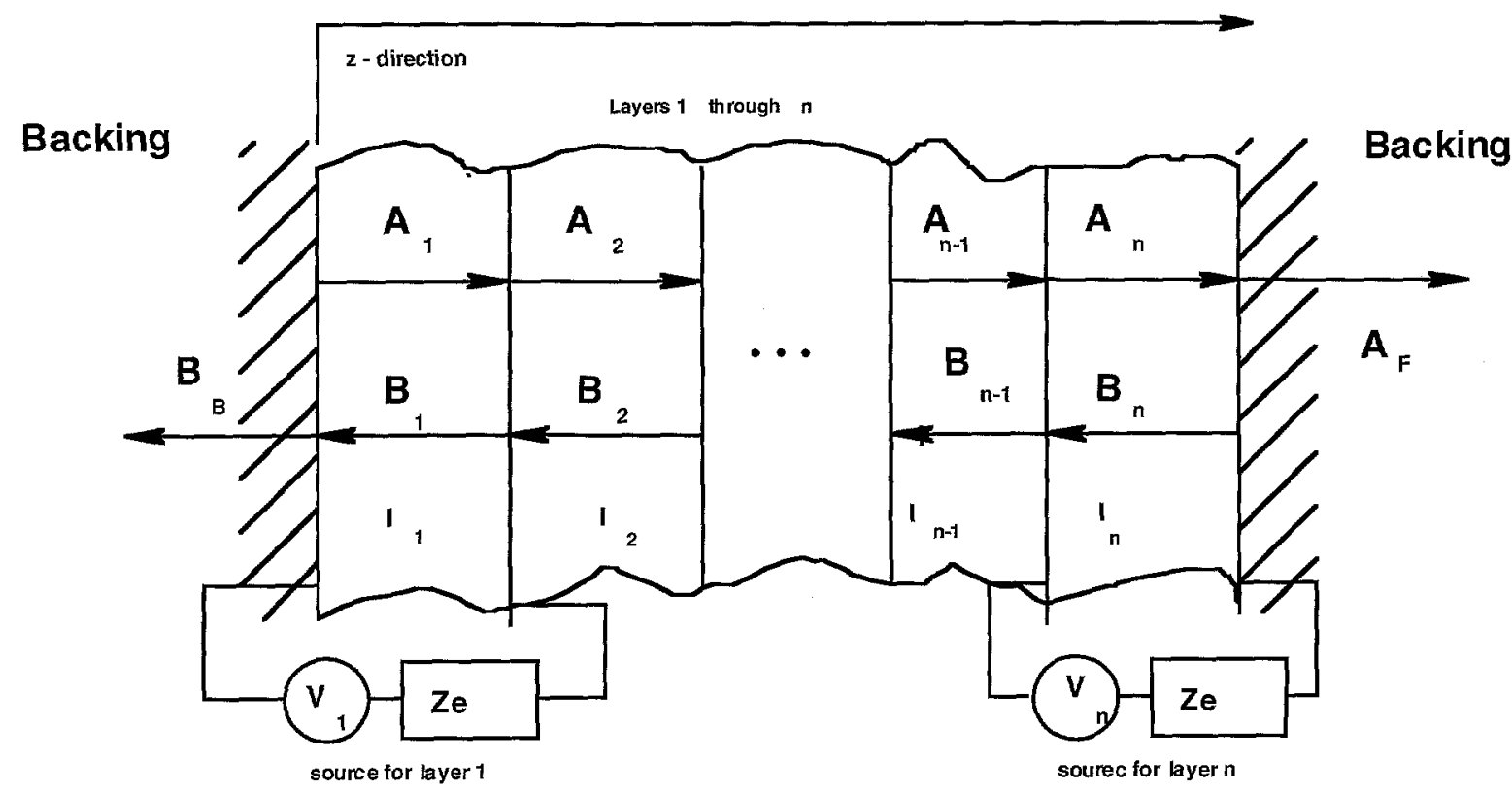

Figure 6. A One-dimensional n-layered system. The A and B coefficients denote the displacement amplitude functions for each layer.

A Laplace domain technique for evaluating the transmission characteristics of a transmitter/ receiver has been presented by Lewis[15]. For our analysis we will follows a similar approach using a Fourier domain solution technique. The one-dimensional wave equation for particle displacement, $\xi_{\mathrm{n}}$, in a given layer is given by

$$
\frac{\partial^{2} \xi}{\partial t^{2}}=v^{2} \frac{\partial^{2} \xi}{\partial z^{2}}
$$

where the $\mathrm{v}$ is the sound speed of the material layer determine from the relevant elastic constant ,$\lambda$, and the density, $\rho$. The solution in the Fourier domain is

$$
\xi(z, t)=\left(A_{n} e^{-i k_{n} z}+B_{n} e^{i k_{n} z}\right) e^{i \omega t},
$$


where the $A_{n}$ and $B_{n}$ are displacement amplitude factors to be determine by a consistent application of the relevant boundary conditions. The Fourier operator is $k$. The relevant boundary conditions are given in terms of stress and velocity on the outer most interfaces

$$
\begin{gathered}
V_{z}(0)=-V_{0}=B_{B}, \\
V_{z}\left(l_{n}\right)=+V_{n}=A_{F}, \\
T_{z}(0)=-P_{\text {rad }}^{o}=-\rho_{B} c_{B} V_{z}(o)=Z_{B} B_{B}, \\
T_{z}\left(l_{n}\right)=-P_{r a d}^{n}=-\rho_{F} c_{F} V_{z}\left(l_{n}\right)=-Z_{F} A_{F} .
\end{gathered}
$$

The specific acoustic impedance of the backing materials is denoted by $Z_{B}$ and $Z_{F}$. Following Lewis [15], the total stress, $T$, on an interface between two layers is given as

$$
T_{3}=c_{3}^{D} S_{3}-h_{33} D_{3}
$$

The second term represents the piezoelectric effect where, $D$, is the electric displacement in the piezoelectric layer and $h$ is a piezoelectric coefficient relating change in charge to stress. Note, the subscript 3 refers to the z-component. Electric displacement is more conveniently represented in terms of the total free charge on the electrode surfaces $Q=D A_{s}$ where, $A_{s}$ is the electrode area of the piezoelectric layer. For non-piezoelectric materials this second term is omitted. The current $I$, is simply $I=d\left(D_{s}\right) / d t$. Also, the linear strain in the direction of propagation is $S_{3}=$ $d\left(d\left(V_{z}\right) / d z\right) d t$. Thus for a single frequency, $\omega$, Eq. (9) may be expressed as

$$
T_{3}=\frac{c_{3}^{D}}{i \omega}\left(-i k_{n} A_{n} e^{-i k_{n} z}+i k_{n} B_{n} e^{i k_{n} z}\right)-h_{33}\left(\frac{I}{i \omega A_{s}}\right)
$$

The voltage in a given layer is obtained from

$$
E_{3}=-h_{33} S_{3}+\left(1 / \varepsilon_{33}^{s}\right) D_{3},
$$

where, $\mathrm{E}$ is the electric field and $\varepsilon$ is the dielectric permeability of the piezoelectric material. For a constant electric field between parallel surfaces, the electric field can be represented in terms of a voltage across the thickness, $t$, of the piezoelectric layer (i.e., $E=V / t$ ). Therefore the voltage across a given layer is

$$
V_{3}^{n}=-h_{33}\left(\xi\left(l_{n-1}\right)-\xi\left(l_{n}\right)\right)+\left(\frac{Q}{C_{o}}\right)
$$

where the capacitance $C_{0}$ of the layer is $\left(A_{s} \varepsilon / t\right)$. The $e^{i \omega t}$ dependence in Eqs. 10 and 11 has been factored out. Applying the boundary conditions (5) through (8) to Eqs. (10) and (11) results in a $(2 n+2)$ by $(2 n+2)$ system of linear equations. The details of which can be found in [15]. The resulting system of equations is show in Figure 7. 


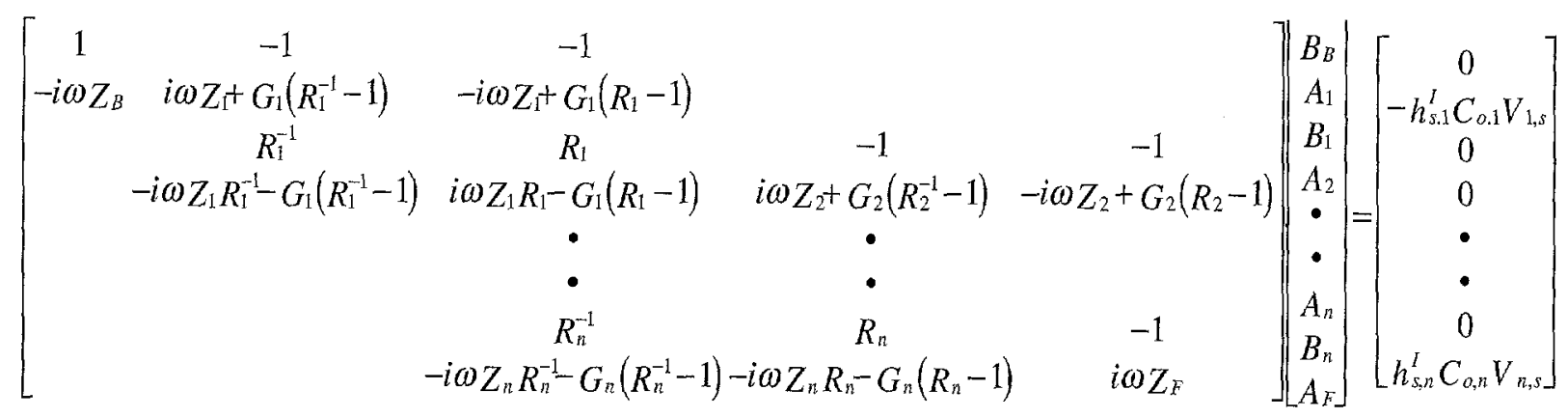

where

$$
\left.\begin{array}{l}
h_{S i}=h_{i} /\left(1+i \omega C_{o i} Z_{e i}\right) \\
G_{i}=h_{h} h_{S i} C_{o i} \\
R_{i}=e^{i k k_{i}}
\end{array}\right\}, i=1, n
$$

Figure 7. After Lewis, [15] and Hossack [16], the system of equations for the nlayered system defined in Figure 6. Typically the known quantities are the backing impedances $Z_{B}$ and $Z_{F}$ the drive voltages $V_{n, S}$, of the piezoelectric layers ( 1 and $n$ ). The acoustic impedance of the layer $n$ is $Z_{n}$. The elastic, piezoelectric, and geometrical properties of each layer are also known along with the frequency. If require the matrix may be re-arranged.

The matrix equation represented in Figure 3 is similar to that present by Lewis [15] and more recently by Hossack [16]. The drive voltages, frequency, and material properties for each layer, including the piezoelectric properties are be specified in addition to the loading impedances $\left(Z_{F}\right.$ and $Z_{\mathrm{B}}$ ) on the outer most edges. A simple inversion of this matrix yields the displacement amplitudes for the waves in each layer. The pressure and velocity profiles in the channel are determined from $V_{z}(z)=i \omega(\xi(z))$ and $P(z)=Z_{f 1} V(z)$. Where $Z_{f 1}$ is the specific acoustic impedance of the fluid. Substitution of these results into $\mathrm{Eq}(2)$ gives energy density of the standing wave pattern for the specified operating parameters.

The accuracy of this system of equations becomes questionable when the length scales of the device are all on the same order of magnitude. Under these circumstances, the 1D planar assumptions are no longer valid and the interaction between the various three-dimensional eigen modes of the chamber increase the complexity of the standing wave field in the chamber. Three dimensional analytical solutions do exist for simplifies boundary conditions however the utility of these is limited by there simplifications. A complete solution of the problem must rely on using numerical techniques such as finite elements.

\subsection{Application of commercial FEM package ABAQUS}

There as several commercially available finite element packages that have the capabilities to solve acoustically coupled fluid-structure interactions. In particular, is a package called ABAQUS from Hibbitt, Karlsson, and Sorrensen, Inc. Numerous references for this code can be obtained from the company web page and the open literature [17]. Our motivation to use this code is based on the need to completely model the dynamic response of the structural-acoustic system to corroborate experimental observations. In addition, we are also interested in testing the validity of the one-dimensional approximation developed in the previous section. Specific details of the model can be found in the literature [17] but a description of a generalized model geometry and system of equations is now be given. 


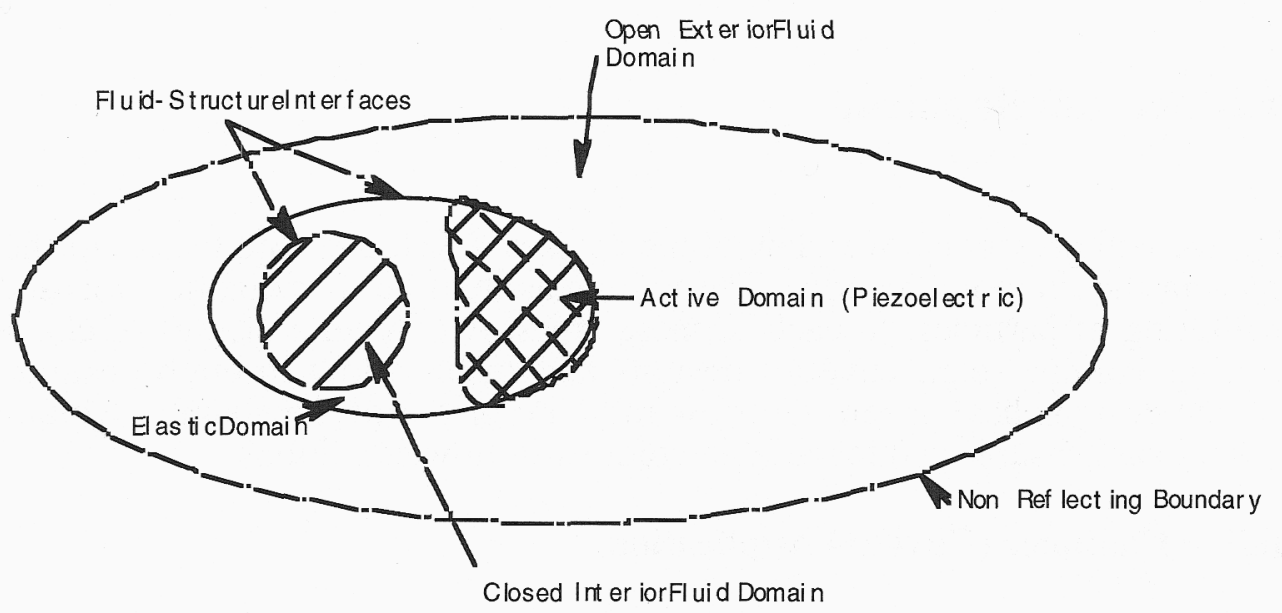

Figure 8. The illustration depicts a generalized mathematical domain that applies a wide range of acoustically coupled fluid loaded elastic and piezoelectric structures.

To model a radiating elastic or piezoelectric structure using finite elements, the mesh must contain the structure as well as the fluid domain. The unknowns are the nodal values of the displacement field $\{U\}$ in the whole structure, the electric potential $\{\Phi\}$ in the piezoelectric material and the pressure $\{\mathrm{P}\}$ in the fluid. The equations represented are based on elastic relations in the structure, Poisson's equation in the piezoelectric material, and Helmholtz's equation in the fluid. Continuity relations are prescribed on the interfaces between the fluid and the structure while appropriate radiation constraint is maintained on the surface surrounding the fluid domain (radiation problems). In matrix form the complete system of equations is written as

$$
\left.\mid \begin{array}{ccc}
{\left[K_{m m}\right]-\omega^{2}[M]} & {\left[K_{u \Phi}\right]} & -[L] \\
{\left[K_{u \Phi}\right]^{T}} & {\left[K_{\Phi \Phi}\right]} & 0 \\
\rho^{2} c^{2} \omega^{2}[L]^{T} & {\left[K_{m m}\right]} & {[H]-\omega^{2}\left[M_{l}\right]}
\end{array}\right]\left[\begin{array}{l}
\frac{U}{\Phi} \\
P
\end{array}\right]=\left[\begin{array}{c}
\frac{F}{-Q} \\
-\frac{Q}{2} \Psi
\end{array}\right] .
$$

Where $\{\mathrm{F}\}$ is the vector of nodal values of the applied forces, $\{\mathrm{q}\}$ is the vector of nodal values of the electrical charge, $\{\Psi\}$ is the vector of nodal values of the integrated normal derivative of the pressure on the boundary $\mathrm{S}$. The mass, stiffness, piezoelectric and dielectric matrices are $[\mathrm{M}]$, $\left[\mathrm{K}_{\mathrm{mm}}\right],\left[\mathrm{K}_{\mathrm{u} \Phi}\right]$, and $\left[\mathrm{K}_{\Phi \Phi}\right]$ respectively. The fluid stiffness and mass matrices are $[\mathrm{H}]$ and $\left[\mathrm{M}_{1}\right]$ and $[\mathrm{L}]$ is a coupling matrix at the fluid interface (connectivity). The fluid density is $\rho$ and the sound speed is $\mathrm{c}$. The frequency is $\omega$. Specific operation and use of the ABAQUS code can be found in the user manuals and will not be presented here. 


\subsection{Hardware development}

In this section we will present an overview of the devices developed with the aid of the modeling tools presented in the previous section. The two areas that the investigation focused on were, mixing and filtering of particles. In each of these areas, two general classes of chambers were studied; standing volume (e.g., cuvette) designs and in-line flow through designs (flocels). Standing volume designs have a zero net flow rate into or out of the chamber volume whereas the flocel devices can be subjected to variable volumetric flow conditions.

\subsection{Standing Chamber (Cuvette) Application:}

In this application the interest is in achieving controlled mixing and focusing of particle in a standing chamber environment. We have also demonstrated the ability to ultrasonically mix fluid samples in micro fabricated chambers. Macro-scale mixing experiments in an ultrasonic bath show that acoustic mixing results in faster antibody binding.

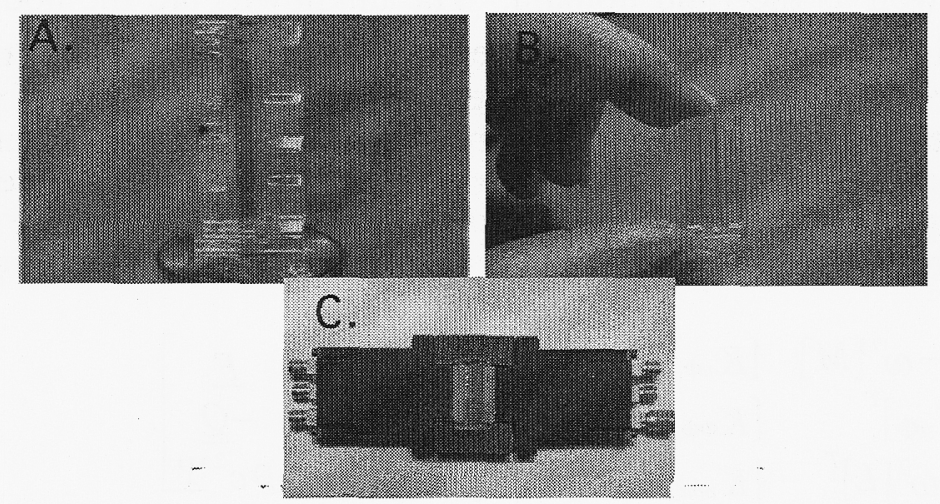

Figure 9. Examples of standing chamber mixing and focusing devices. Chamber volume approximately 200 ul. See text for details.

Figure 9 shows two different standing chamber designs. The device on the left (chanber.A), is for mixing while the device on the right, (chamber B), can be operated in either a mixing or focusing mode. A complete package with wiring connections is shown in Fig. 8.C. The devices shown are constructed from acrylic although later designs have shown better performance using glass. The fluid volume of these chambers is approximately 200ul. Mixing in chamber A is obtained from 4 PZT drivers (not shown) driven at a set frequency but located at non-symmetrical locations on the channel walls. The drivers create a non stable acoustic field which induces mixing. The device on the right (chamber B) uses two symmetrically located drivers. Device B can be made even simpler by careful design using only a single PZT driver and relying on internal reflections from the opposite wall to create a standing wave field. Mixing in device B is achieved by choosing an appropriate drive frequency that excites two different channel modes. These two different modes interact to create an unstable standing wave pattern that promotes mixing of the contained fluid. This effect is not unique to the B device. Similar behavior exists in the 4 driver design, this effect however is secondary to the primary instability created by the offset geometry. 


\subsubsection{One-dimensional transmission matrix approximation}

The ability to easily predict the performance of a particular channel design was the motivation for the one-dimensional approximation developed in Section 3. Figure YZ shows the analysis geometry. The layered system is basically a one-dimensional approximation to the real device (chamber B, Figure 9). The device uses two opposing piezoelectric transducers (PZT 4) denoted as layers 1 and 5 that are coupled directly to an acrylic chamber filled with water. Similar operation has been obtained by eliminating one of the driving elements. In this configuration, the standing wave pattern is created by superposition of a transmitted wave with its reflection off the opposite wall of the chamber.

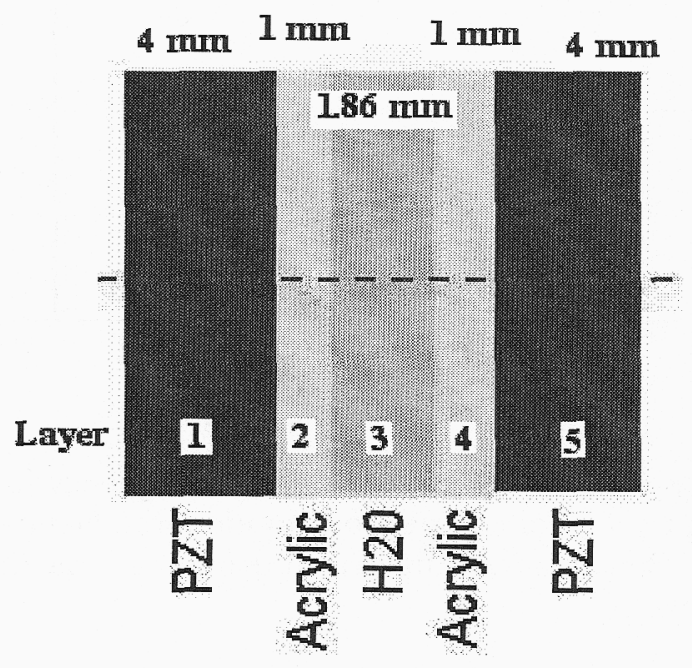

Figure 10. The illustration shows a one-dimensional approximation for a type B standing wave chamber pictured in Figure 9. Channel dimensions and materials are typical. The actual device is modeled with a system that has $n=5$ layers.

The fundamental parameter that measures the particle control performance for a given device geometry is the energy density described by Eq. (2). This expression can be simplified by realizing that for planar one-dimensional waves, the acoustic pressure, $p$, and velocity , $u$, are related through the specific acoustic impedance (i.e., $p=Z_{a} u$ where $Z_{a}=\rho c$ and $\rho$ and $c$ are the fluid density and speed of sound respectively). Thus for planar waves, the energy density in the channel becomes

$$
E_{a c}=\frac{1}{2} \rho_{o} u^{2}=\frac{1}{2} \rho_{o} \omega^{2}\left(A_{3}+B_{3}\right)^{2}
$$

where the displacement amplitude coefficients in the fluid are $A_{3}$ and $B_{3}$ and determined from Eq (12). The utility of this model is realized when one evaluates Eq. 14 as a function of frequency for a particular channel design. The ability to target specific frequencies that maximize the energy density in the channel is very useful for accelerating the design process and maximizing the acoustic control. A typical example is shown in the graph below. Evident from these results is that there are specific frequencies at which the energy density is at a maximum. 


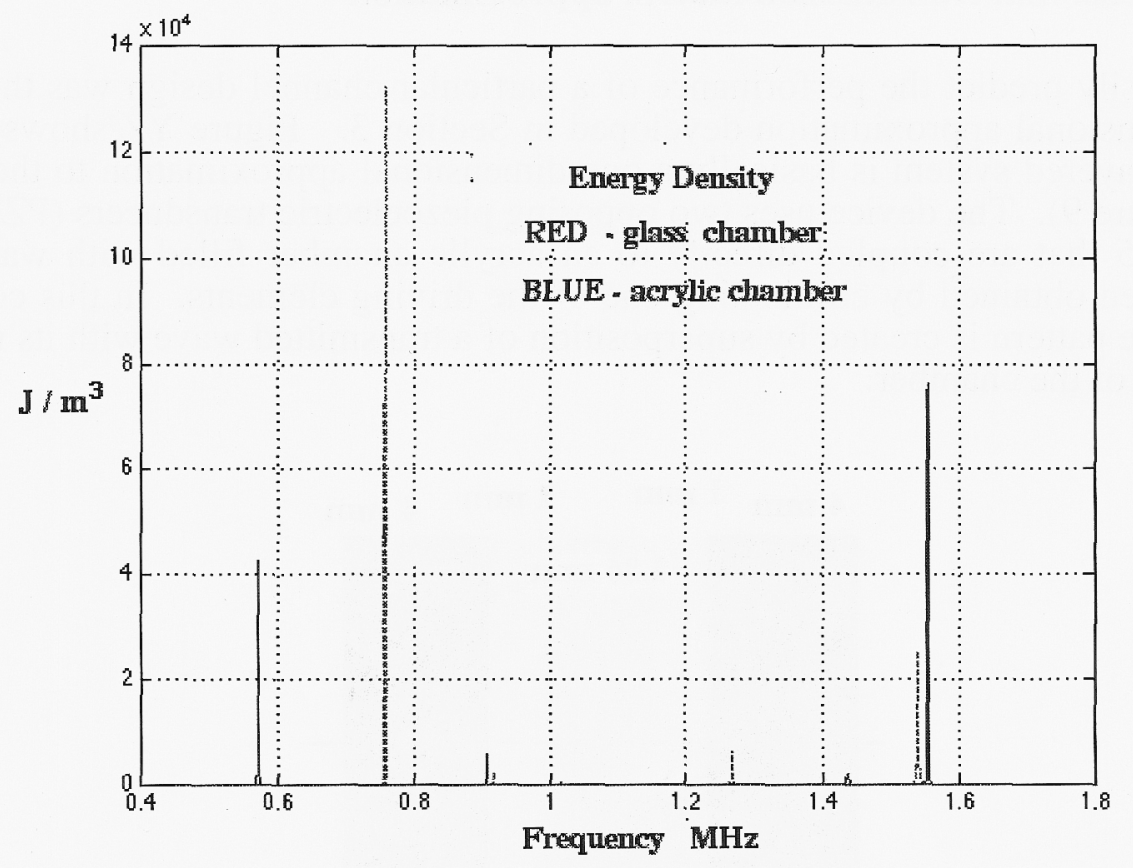

Figure 11. Energy density (Eq 14) is calculated vs drive frequency for the acrylic channel (channel B) shown in Figure 9. For comparison, an identical channel that is constructed from glass is also shown. Both channels are driven using the same drive voltage (20Vpk$\mathrm{pk)}$ on the piezoelectric layers.

The greatest of these maxima (approximately $13 \mathrm{~kJ} / \mathrm{m}^{3}$ ) occurs at $763 \mathrm{kHz}$ in the glass chamber. Similarly, the largest energy density for the acrylic chamber (approximately $8 \mathrm{~kJ} / \mathrm{m}^{3}$ ) occurs at $1.56 \mathrm{MHz}$ or slightly larger than twice the glass chamber. Neither of these frequencies nor any of the other calculated maxima are near the intended operational frequency of $0.4 \mathrm{MHz}$. This frequency was initially selected using geometrical arguments (i.e., $f=c /(\lambda)$, where $\lambda=2 d$ and $d$ is the channel width). The intent was to create a single half wavelength pressure field with a node in the center of the channel. From a performance standpoint, this is not an optimal frequency to operate the channel at.

Once the frequencies at which the maximum(s) of energy density function occurs at are determined, the pressure and velocity profiles these frequencies provide insight into the number and location of nodes or anti-nodes in the channel. Ultimately it is these nodal locations where different particle species will collect depending upon their "compressidensity" function. Unfortunately, applying this model to devices that have strong two- and three-dimensional characteristics can lead to erroneous results. Therefore this approximation is used in conjunction with finite element models to accurately determine channel performance and behavior.

\subsubsection{Two-dimensional ABAQUS finite element model}

Design and development of the standing wave chambers was augmented by application of a commercial finite element package (ABAQUS). Initial modeling efforts were focused on developing two-dimensional model of the standing chamber. See Figure 12. The mesh geometry, material and piezoelectric constants, and drive voltages are identical to those described above. The length of the channel is approximately $26 \mathrm{~mm}$. The following figure shows the two-dimensional finite element model and corresponding laboratory images of the 
actual device in operation. In conjunction, the one-dimensional approximation for optimal energy density for these frequency ranges is also shown.

A. 2D finite element model (ABAQUS)

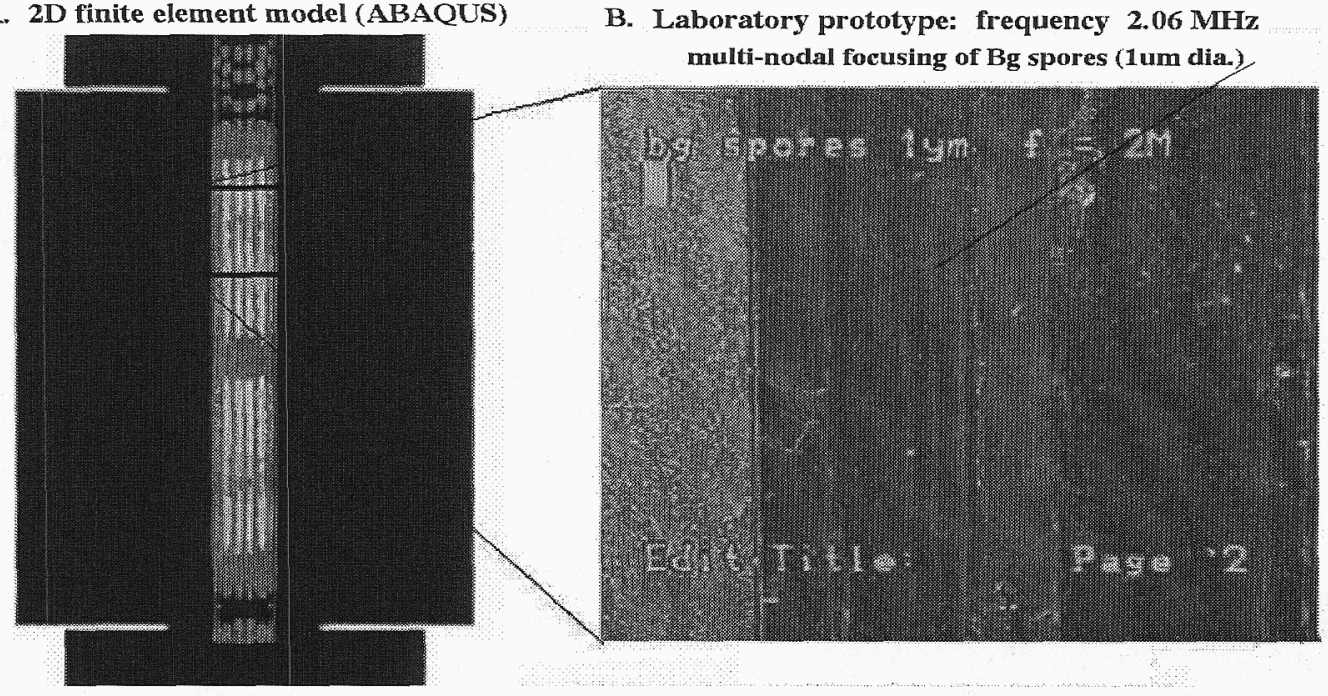

C. $1 \mathrm{D}$ transmission matrix (design guideline)

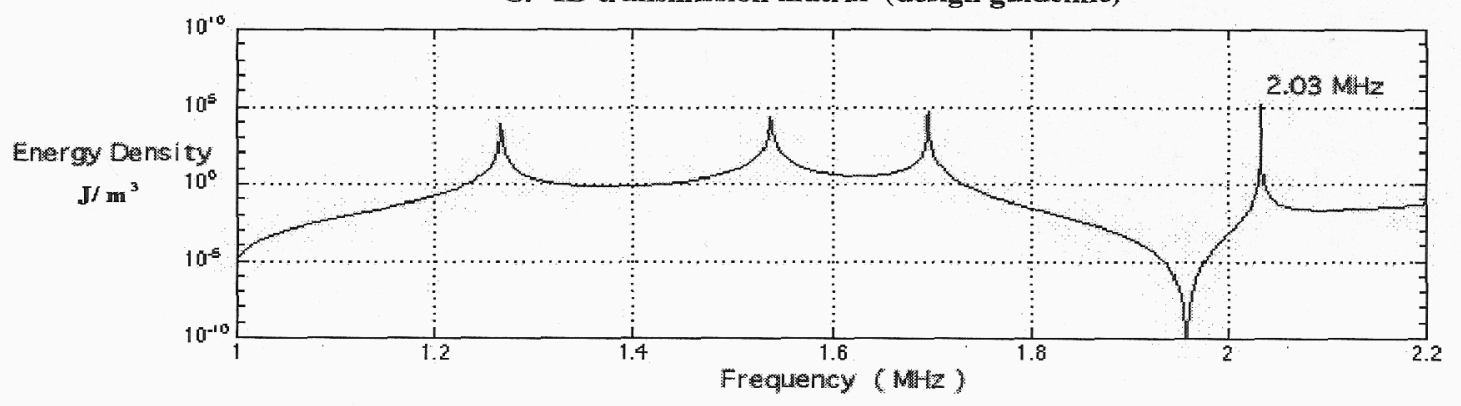

Figure 12. Standing wave chamber. Figure 12.A.shows the results from the two-dimensional ABAQUS model (drive frequency $2.03 \mathrm{MHz}$ ). The chamber and piezoelectric elements are shown in dark blue. The fluid region is in the center where pressure nodes are denoted as light blue along the fluid channel. Figure 12.B shows a view from a video microscope showing the focusing of $\mathrm{Bg}$ spores ( 1-2um dia) at the corresponding pressure nodes predicted by the finite element model. Finally, Figure 12.C is a plot of channel energy density as a function of frequency calculated by the one-dimensional transmission matrix model. The maximum energy density is estimated to be at $2.03 \mathrm{MHz}$.

Based on the results from the transmission matrix model, there are several other optimal frequencies that the channel can be driven at (i.e., $1.25 \mathrm{MHz}, 1.55 \mathrm{MHz}$, and $1.7 \mathrm{MHz}$ ). Each of these frequencies was investigated in the laboratory using the same drive voltages specified at $2.06 \mathrm{MHz}$. The expected performance was only realized at the $1.7 \mathrm{MHz}$ peak. The other peaks either failed to focus the $\mathrm{Bg}$ spores or mixing was induced. Simulations from the twodimensional ABAQUS model revealed complicated standing wave fields at these other frequencies suggesting that there are significant two-dimensional effects that the onedimsnsional approximation is neglecting. 
Our results have found that the largest peaks in the energy density function correspond to frequencies for which the channel width is in the range $(\lambda / 2<d<2 / 3 \lambda)$. At these frequencies, the energy density is so large that structural modes are being excited resulting in violent mixing and even cavitation

\subsubsection{Mixing (enhanced binding)}

Mixing fluids in microscale devices is challenging because of the laminar flow conditions, yet mixing is an essential function for performing bioassays. Mixing by diffusion is slow, with diffusion rates on the order of one $\mathrm{mm}$ in 20 minutes. Devices such as DNA chips require significant time allowances for diffusion and interaction of the DNA strands with different areas on the chip; otherwise, false negatives might result. The same is true for NBC-based assays. Detuning the transducers in the acoustic device shown in Figure 9 provides effective mixing (see Fig. 13A). In addition, we have shown that acoustic excitation enhances binding kinetics of fluorescent tags to surface-functionalized beads by up to $20 \%$ (Fig. 13B).
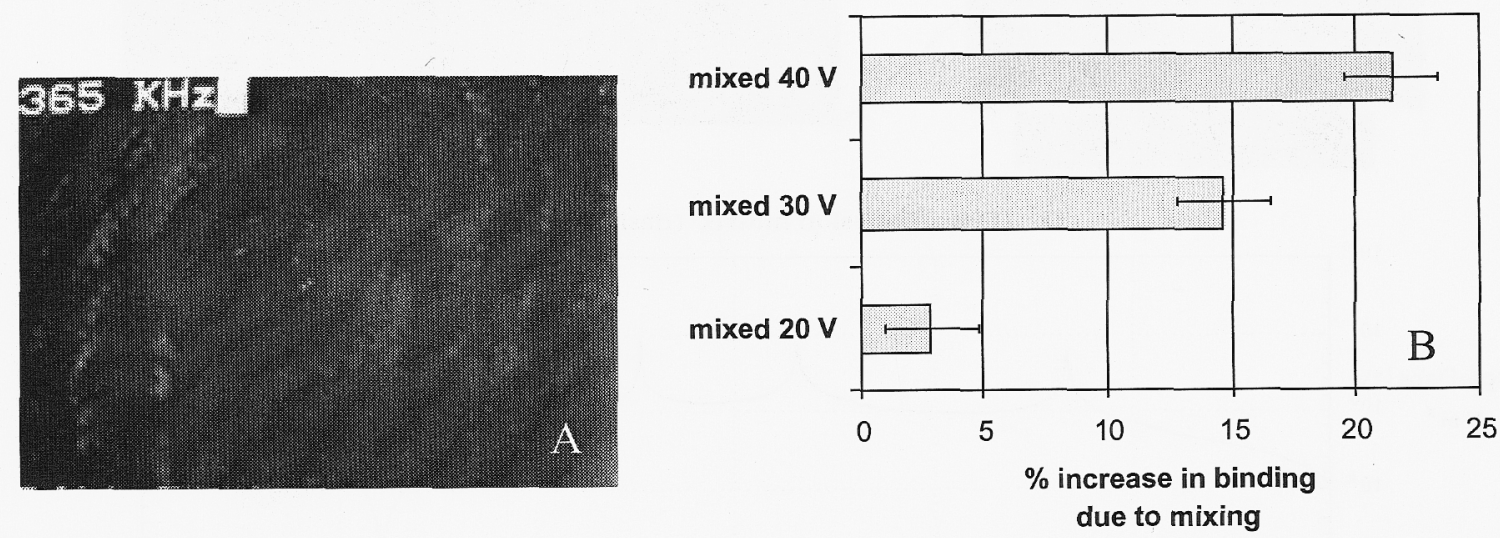

Figure 13. Mixing in a microfluidic device by acoustic forces (A). Swirling beads are 10 microns in diameter. Data showing percentage increase in fluorescence tag binding due to acoustic mixing at different voltages (B). 


\subsection{Flow -through device (flocel) application:}

The Flocel device is an adaptation of the Standing wave described in the previous section. This device is constructed from bonding three separate glass layers to form an enclose cavity. Two small inlet and exit ports (approx. $0.5 \mathrm{~mm}$ dia.) are at either end of the channel. The design of this device was motivated by applications requiring non-contact manipulation of particles flowing in an ambient fluid suspension. The chamber geometry and material properties are similar to the standing wave chamber. The flocel designs rely only on a single piezoelectric driver to create the standing wave pattern. This simplifies the overall construction and electrical control of the device. The optimization of the channel design followed the same process use for the standing chamber. The FEM mesh for the ABAUQS finite element calculations are shown in Figure 14. The flocel is represented in three different ways, perspective, mesh and an internial view of the standing wave chamber
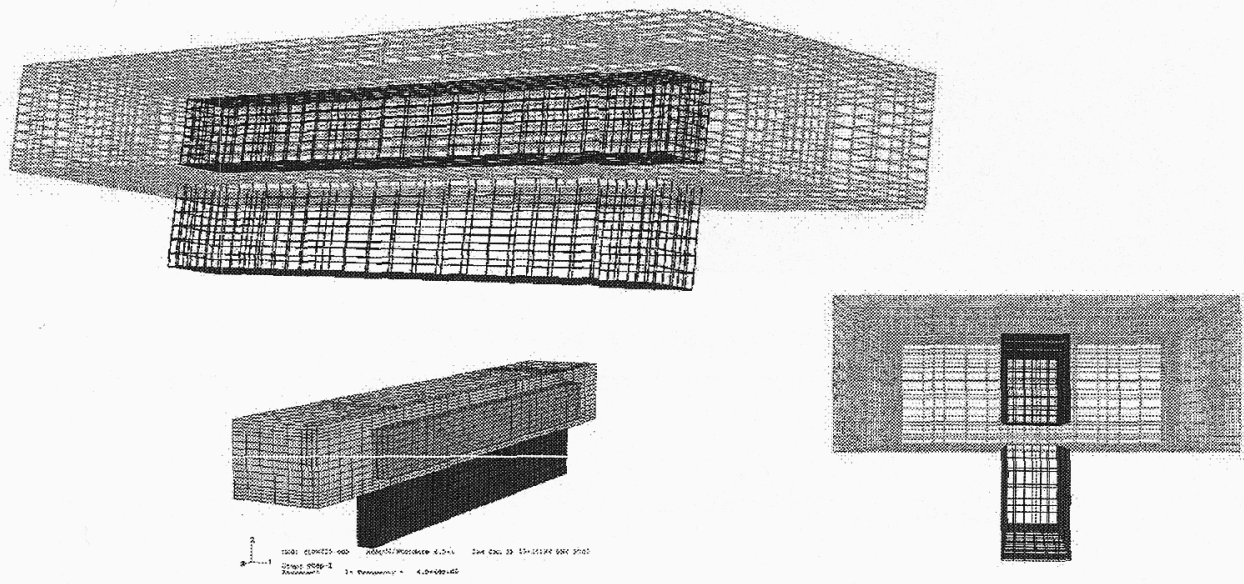

Figure 14. The current flocel geometry is shown in two different views; a mesh outline and a solid model with half of the glass chamber removed. The glass chamber is shown in green or yellow, the fluid is in blue and the piezoceramic element is brown.

Results of the 3D finite element model, the corresponding one-dimensional approximation and the actual device in operation are illustrated in Figure 15. The transmission matrix model has targeted several candidate frequencies for maximizing the acoustic energy density in the channel. Of the six peaks shown, only two provided stable standing wave patterns that resulted in particle focusing in the flocel device; these were $1.57 \mathrm{MHz}$ and $1.66 \mathrm{MHz}$. The remaining maxima created varying degrees of mixing conditions in the channel. The cause of this mixing is uncertain, however, the same line of thinking presented for the standing wave chamber is also true here. It is possible that the acoustic energy in the channel is exciting wall resonances in the chamber which are resulting in unstable standing wave fields. Also, the driving elements exhibit multi-nodal strain distributions that would in turn create non-planar excitation condition on the wall of the chamber where the piezoelectric driver is mounted. This non-planar excitation will result in a non-uniform boundary condition on the fluid in the chamber that can contribute unstable pressure fields and induce mixing. Further work is necessary to understand the complicated phenomena present in these types of contained sound fields. 


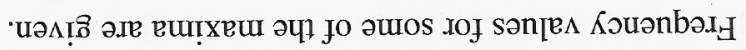

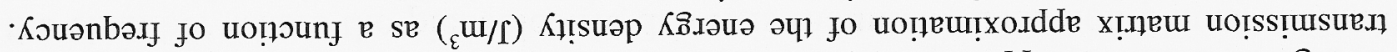

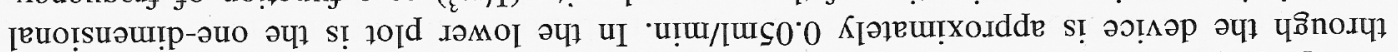

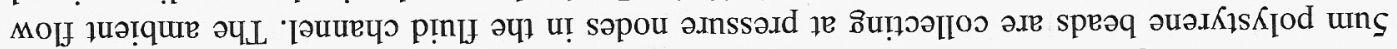

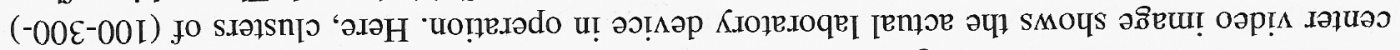

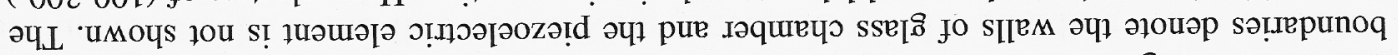

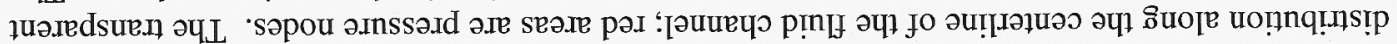

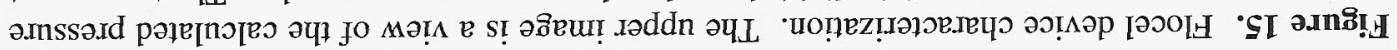

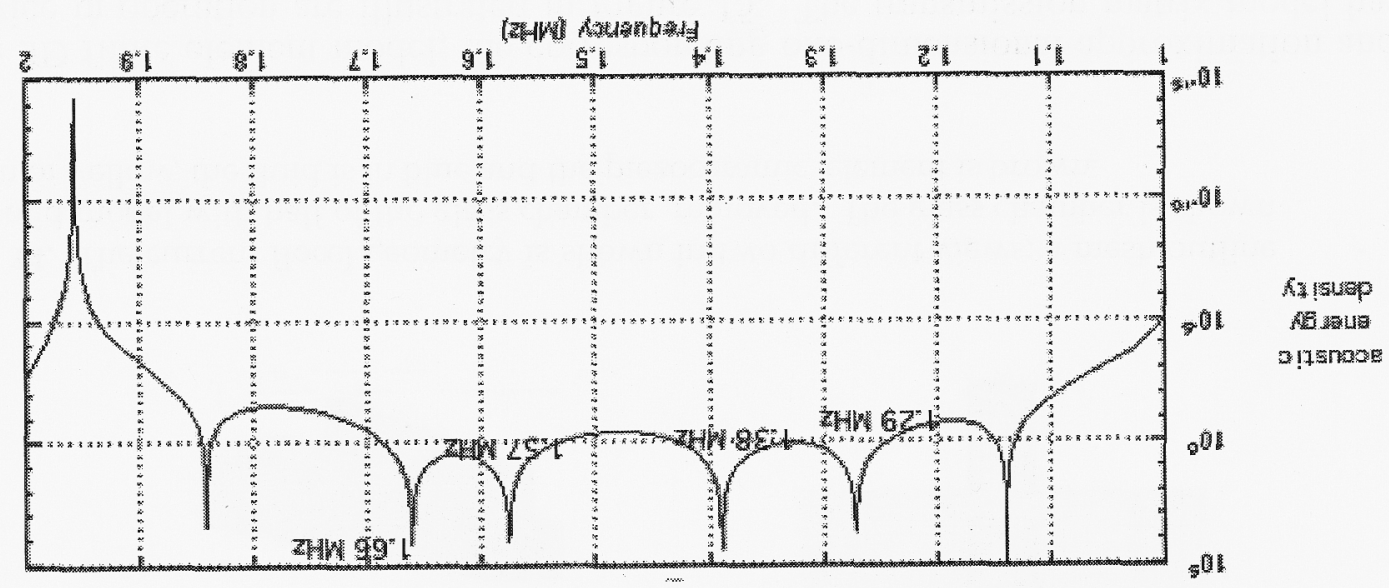

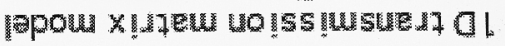

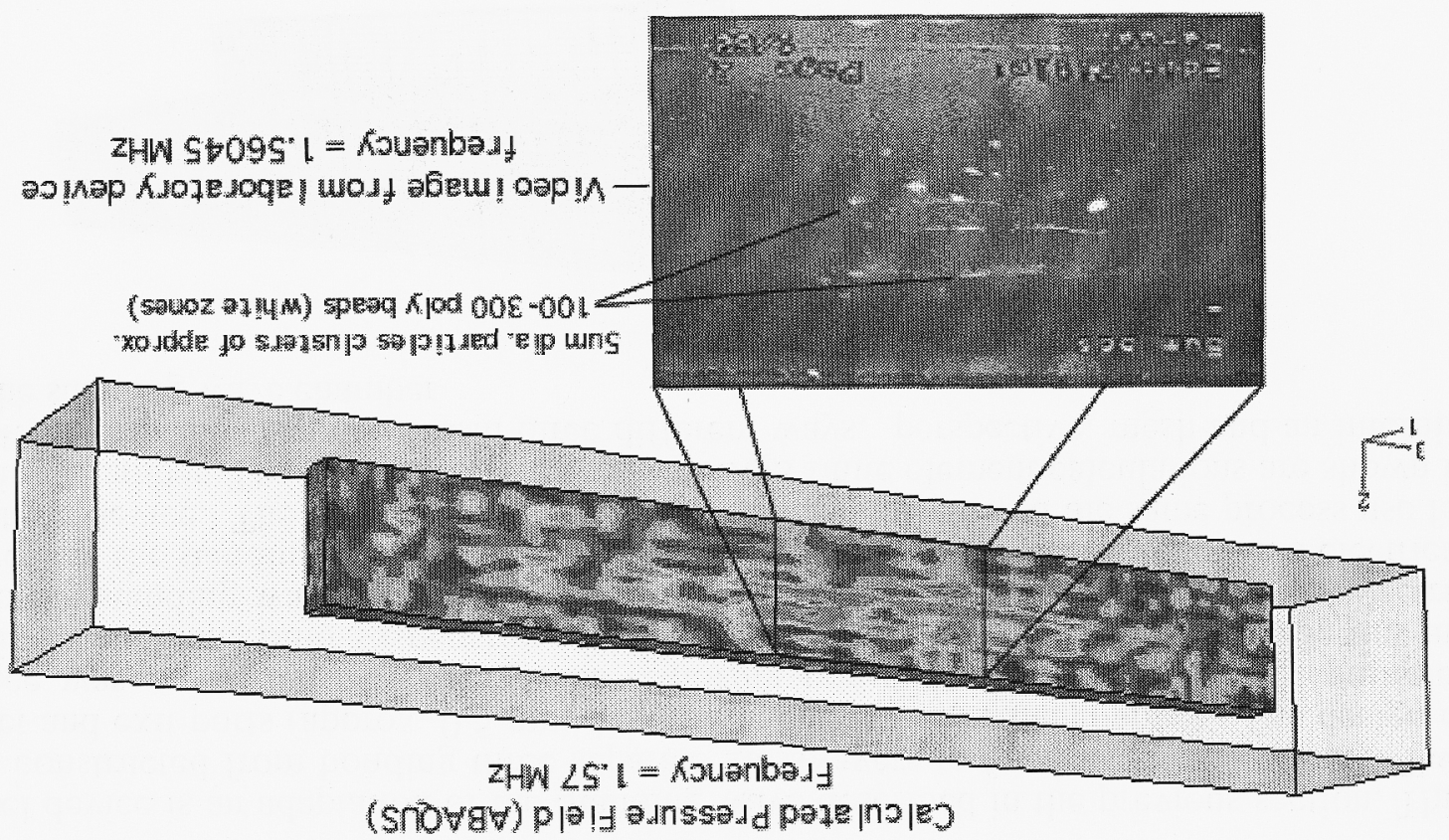




\subsubsection{Holding/Filtering of particles in flowing medium}

One application of the flocel chambers is the ability to filter or trap particulates in an ambient flow. Figure 16 show LLNL has used a device similar to the one shown in Figure 14 to trap SurroMed Nanobarcodes (NBCs) in a flowing stream, as shown in Figure 15. Fluid was flushed through the system, replacing the existing fluid, while the nanobarcodes were held in place by acoustic forces. In this case, the nodal pattern was more complex than a simple standing wave. The chamber of the microfluidic device was $0.4 \mathrm{ml}$ and the flow rate was $0.8 \mathrm{ml} / \mathrm{min}$.
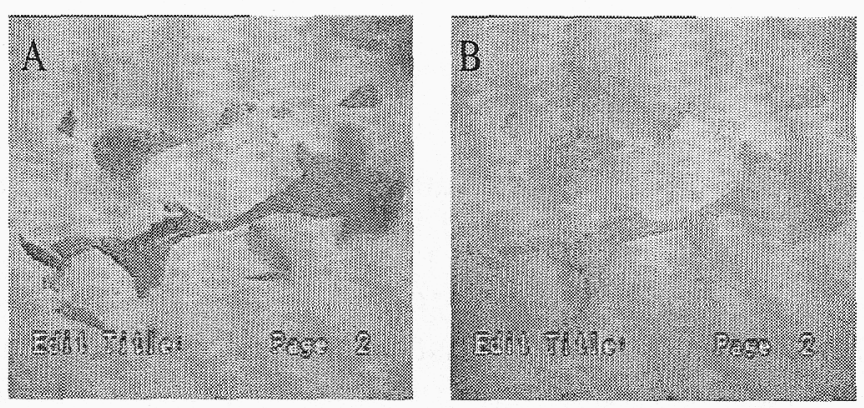

Figure 16. Trapping and washing of NBCs in a microfluidic device. The NBC's are trapped using acoustic radiation forces and a dyed fluid is flushed through the system at $0.8 \mathrm{ml} / \mathrm{min}$. The color change from flushing with the dyed solution is evident from (A) to (B).

This effect can be used to replace a centrifuging operation to enable miniaturization and automation of manual sample preparation procedures, and may be incorporated into the proposed Nanobarcode microfluidic module. The data in Figure 14 show the relationship between flow rate and particle retention for acoustic trapping experiments with $6 \mathrm{um}$ diameter particles. At flow rates as high as $0.1 \mathrm{ml} / \mathrm{min}, 87 \%$ of the particles were retained within the system.

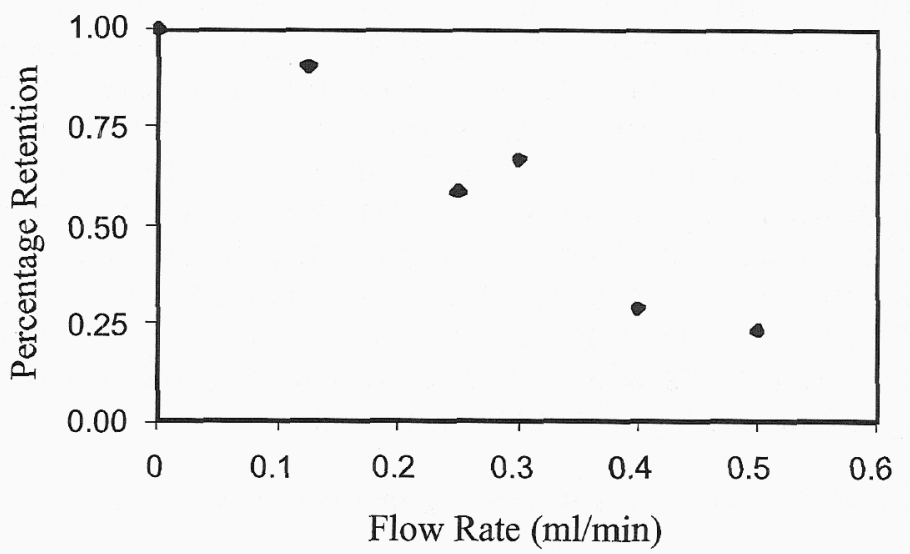

Figure 17. Percentage particle retention vs. flow rate for 6 um diameter beads trapped by acoustic radiation pressure. 


\subsection{Commercialization of selected results}

The results of this LDRD project have caught the attention of two biotech companies. The first, CytoLogix Inc., is interested in commercializing the microfluidic mixing techniques to enhance hybridization on glass microscope slides. The second company SurroMED, is developing a micro-particle technology call Nanobarcodes ${ }^{\mathrm{TM}}$ these particles are rod shaped with an approximate diameter of $1 \mathrm{um}$. Both companies have a strong interest in the development of techniques and process the facilitate manipulation of small particles or mixtures of particles and solutions.

\section{Cytoliogix WFO: mixing of dissimilar fluids}

CytoLogix is currently developing an instrument to completely automate nucleic acid hybridization on glass microscope slides. The instrument will be used to perform complex DNA/RNA hybridization on tissue sections and DNA microarrays. Currently, the majority of hybridizations are performed manually using $\sim 20 \mathrm{uL}$ of probe solution under a glass coverslip. Semi-automated hybridization instruments exist in the marketplace, but there are no fully automated instruments that meet the challenges of DNA hybridizations. The key challenges to developing an automated hybridization instrument are:

- Low probe volume.

- $\quad$ Mixing to provide uniform specificity and uniform staining intensity.

- Uniform heating of the specimen and probe solutions.

- Innovative clamp mechanism to achieve consistent force levels for sealing the chamber without breaking the glass slide.

A need exists to develop a non-contact mixing technology that can be used with the microchamber technology to provide uniform specificity and intensity of the stained specimens by moving the DNA probes (in solution) around the tissue sample or microarray during the hybridization step. An additional feature of mixing during hybridization is decreased hybridization times resulting in significant cost savings to end users.

LLNL and CytoLogix Inc. are currently in the process of developing a work for others (WFO) agreement that will be a basis for the development of an automated slide processing instrument. Central to this partnership is the fluid mixing module to be jointly developed by CytoLogix and LLNL. In the proposed work, LLNL will perform fundamental studies to develop a robust costeffective method to induce fluidic mixing in a sample chamber. Fluid mixing will be accomplished by using acoustic energy coupled from external transducers into the fluid chamber. Adaptation of commercial designs or LLNL designed and fabricated devices will be used. Multiple designs will be modeled and tested with the final goal of developing a proof-ofprinciple prototype. The prototype will be used as a test bed for mixing and response measurements. Prototype instruments will be delivered to CytoLogix for further testing, design refinements, and integration into the CytoLogix' instrument.

\section{SurroMED Nanobarcodes: Non-contact particle manipulation}

A proposal is being submitted to NIH by Peter Krelevitch and Dennis Polla which will partially rely on some of the non-contact particle manipulation techniques presented this LDRD. The goal is to develop an integrated microfluidic module for performing multiplexed cancer marker assays based on SurroMed's Nanobarcodes ${ }^{\mathrm{TM}}$ identification tag technology. Nanobarcodes (NBCs) are rod-shaped nanoscale particles that are surface-functionalized and encoded for optical identification. Thousands of distinct NBCs can be fabricated, enabling multiplexed, flow- 
through detection of molecular biomarkers of cancer, including DNA, RNA, and proteins. This promising technology, combined with microfluidic sample handling capabilities developed at Lawrence Livermore National Laboratory, will enable automated processing suitable for clinical application for early cancer detection, as well as cancer biomarker discovery.

\subsection{Summary}

As mentioned in the introduction, the main objective of this project is concerned with the research and development of a technique(s) to manipulate small particles using acoustic energy coupled into a fluid filled plastic or glass sample chamber. To achieve this goal, we applied a design methodology that encompassed analytical, computational, and experimental methods. Our purpose was to understand, develop, and optimize acoustic filtration, fractionation, and mixing in microfluidic systems. This research has resulted in the following:

- A new understanding of the complex nature of acoustically coupled microfluidic structures.

- $\quad$ Fostered the development of commercial partnerships with LLNL and biotech companies.

- Generated a knowledge base for further research in the area of high intensity acoustic based cell lysing.

\subsection{References}

[1] Whitworth G., Grundy M.A., Coakley W.T., “Transport and harvesting of suspended particles using modulated ultrasound", Ultrasonics (29), p.439-444, 1991.

[2] Gaida Th., Doblhoff-Dier O.,Strutzenberger K., Katinger H., Burger W., Groschel, M., Handl B., Benes E., " Selective Retention of Viable Cells in Ultrasonic Resonance Field Devices”, Biotechnolo. Prog. 1996, (12)1, 73-76.

[3] Yasuda K., Stephan S.H., Umemura S., "Using acoutic radiation force as a concentration method for erythrocytes",J. Acoust. Soc. Am., (102)1, 1997.

[4] Higishitani K., Fuckushima, Y., Matsuno Y., "Migration os suspended particles plane stationary ultrasonic fields", Chem. Eng. Sci. 36(1981) 1187.

[5] L. V. King, Proc. Roy. Soc., A, 147. 212, 1934.

[6] Yosioka, Y. Kawasima, "Acoustic Radiation Pressure on a compressible sphere," Acustica, 5, 167-173, 1955.

[7] K . Yasuda, K Takeda, S. Umemura, "Studies on particle separation by acoustic radiation force and electrostatic force," Jpn. J. Appl. Phys., 35, 3295-3299, 1996.

[8] Chen, R., Sitzo, L., Osorio, N.C., Hsu, B., Rodgers, R. Litman D.J., "Ultrasonicaccelerated immunoassay, exemplified by enzyme immunoassay of choriogonadotropin", (1984) Clinical chemistry, (30):1446-1451.

[9] Schmidt, P., Rosenfield, E, Millner, R., Schellenberger, A., "effects of ultrasound on the catalytic activity of matrixed-bound glucoamylase, (1987), Ultrasonics, 25:295-299.

[10] Wang A. W., Kiwan W., White, R. M., Ceriana, R.L., (1998), Sensors and Actuators B, 49 13-21. 
[11] Clague et at., The hydrodynamic permeability of (un) bounded fibrous mesia using the lattice Boltzmann method, 1999, submitted to Physical Review E.

[12] Clegure et al., "The hydrodynamic force and torque of acting stationary spherical bodies in Pioiseuille flow", to be Submitted

[13] Krimholtz, R., Leedholm A., and Matthaei, " new Equivalent circuits for elementary piezoelectric transducers”, Electron Lett., vol. 6, no. 13, pp. 398-399, 1970.

[14] Redwood, M., Transient behavior of a piezoelectric transducer", J. Acoust. Soc. Amer., vol. 33, no. 4, pp. 527-536, 1961.

[15] Lewis, G.K., "A matrix technique for analyzing the performance of multilayered front matched and backed piezoelectric transducers", Acoust. Imaging, no. 8, pp 395-416.

[16] Hossack J. A., Auld B.A., "Improving the Characteristics of a transducer using multiple Piezoelectric layers.", IEEE Trans. Ultrason, Ferro. And Freq Contl. ., vol. 40(2) , pp 131-139. March 1993.

[17] ABAQUS Users Manual, Hibbitt, Karlsson, and Sorensen, Version 6.2. 1999. 